UCRL-ID-138550

\title{
Semiconductor Quantum Dots for Advanced Blue Light Emitting Devices and Laser Diodes
}

Howard Lee

Peter Thielen

April 20, 2000

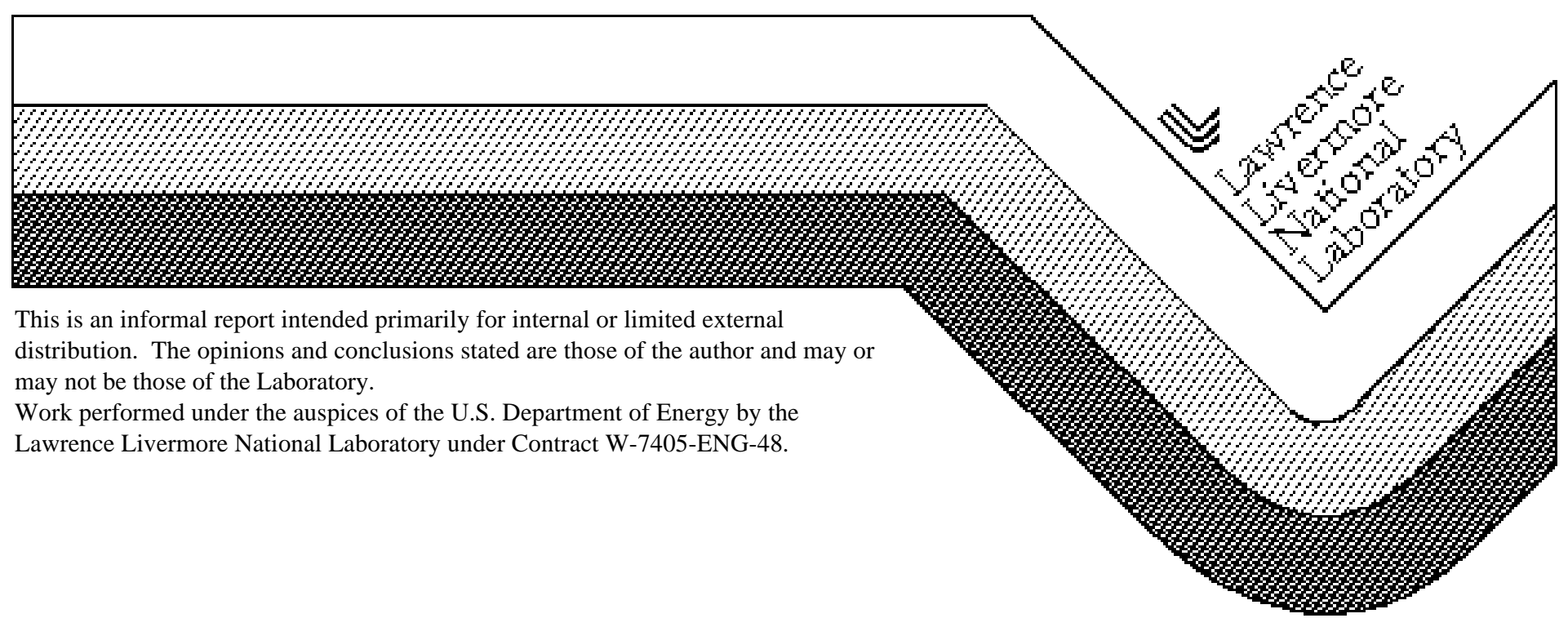




\section{DISCLAIMER}

This document was prepared as an account of work sponsored by an agency of the United States Government. Neither the United States Government nor the University of California nor any of their employees, makes any warranty, express or implied, or assumes any legal liability or responsibility for the accuracy, completeness, or usefulness of any information, apparatus, product, or process disclosed, or represents that its use would not infringe privately owned rights. Reference herein to any specific commercial product, process, or service by trade name, trademark, manufacturer, or otherwise, does not necessarily constitute or imply its endorsement, recommendation, or favoring by the United States Government or the University of California. The views and opinions of authors expressed herein do not necessarily state or reflect those of the United States Government or the University of California, and shall not be used for advertising or product endorsement purposes.

Work performed under the auspices of the U. S. Department of Energy by the University of California Lawrence Livermore National Laboratory under Contract W-7405-Eng-48.

This report has been reproduced directly from the best available copy.

Available to DOE and DOE contractors from the Office of Scientific and Technical Information

P.O. Box 62, Oak Ridge, TN 37831

Prices available from (423) 576-8401

http://apollo.osti.gov/bridge/

Available to the public from the National Technical Information Service

U.S. Department of Commerce 5285 Port Royal Rd., Springfield, VA 22161

http://www.ntis.gov/

OR

Lawrence Livermore National Laboratory Technical Information Department's Digital Library http://www.llnl.gov/tid/Library.html 


\section{SEMICONDUCTOR QUANTUM DOTS FOR ADVANCED BLUE LIGHT EMITTING DEVICES AND LASER DIODES}

98-LW-058

Howard Lee and Peter Thielen

\section{Introduction}

Blue light emitting devices (LEDs) are rapidly becoming an increasingly important technology underscored by intense world-wide research and development. Blue emitter technology is the cornerstone for crucial applications that include full-color flat panel displays, ultra-high density optical memories and data storage, back lighting, and chemical and biological sensing. Currently, the GaN material system dominate the field of blue emitters, which in turn is dominated by Japanese researchers. However, critical obstacles remain for this material system. Growth-related defects, which arise from lattice-matching problems, degrade the device and limits operational lifetimes. Blue GaN diodes produced in Japan presently operate for the longest time. Those produced in the US have significantly shorter lifetimes.

\section{Goals}

Our goal for this project is to expand our knowledge of the fundamental properties of semiconductor quantum dots and to apply this knowledge to the development of advanced blue LEDs and laser diodes that fully exploit the uniquely desirable optical and electronic properties of semiconductor quantum dots. We specifically explored the enhanced light emitting properties of indirect bandgap ( $\mathrm{Si}$ and $\mathrm{Ge}$ ) and direct bandgap ( $\mathrm{ZnSe}$ and $\mathrm{GaN}$ ) quantum dots.

This project involved: (1) investigating the fundamental physics responsible for the unique optical and electronic properties of quantum dots, (2) based on this understanding, designing the appropriate QD-LEDs and QD-lasers that take advantage of these properties, and (4) characterizing and optimizing these devices (efficiency, output spectra, etc.).

\section{Accomplishments for FY99}

Semiconductor quantum dots (QDs) are an innovative and potentially superior alternative to the GaN system for various applications. The properties of QDs are size-dependent due to quantum confinement of electrons and holes as a direct result of their nanometer size. In particular, quantum confinement of carriers causes the energy gap of the QDs to change with size. Thus, QDs enable broad tunability of the output color with a single material system by 
changing the size of the QDs. Different semiconductor QDs can be used to achieve even broader spectral tunability. We have demonstrated for the first time that by changing the size of silicon (Si) or germanium (Ge) QDs, their photoluminescence can be stable in ambient and can be tuned from the red to the ultraviolet. This is illustrated in Figure 1. We also demonstrated for the first time that the size dependence of the quantum dot energy gap can widen over a range much larger than previously thought, as shown in Figure 2. Our results agree with pseudopotential and tight binding calculations, but disagree with effective mass approaches, which is known to over estimate the quantum dot energy at small sizes.

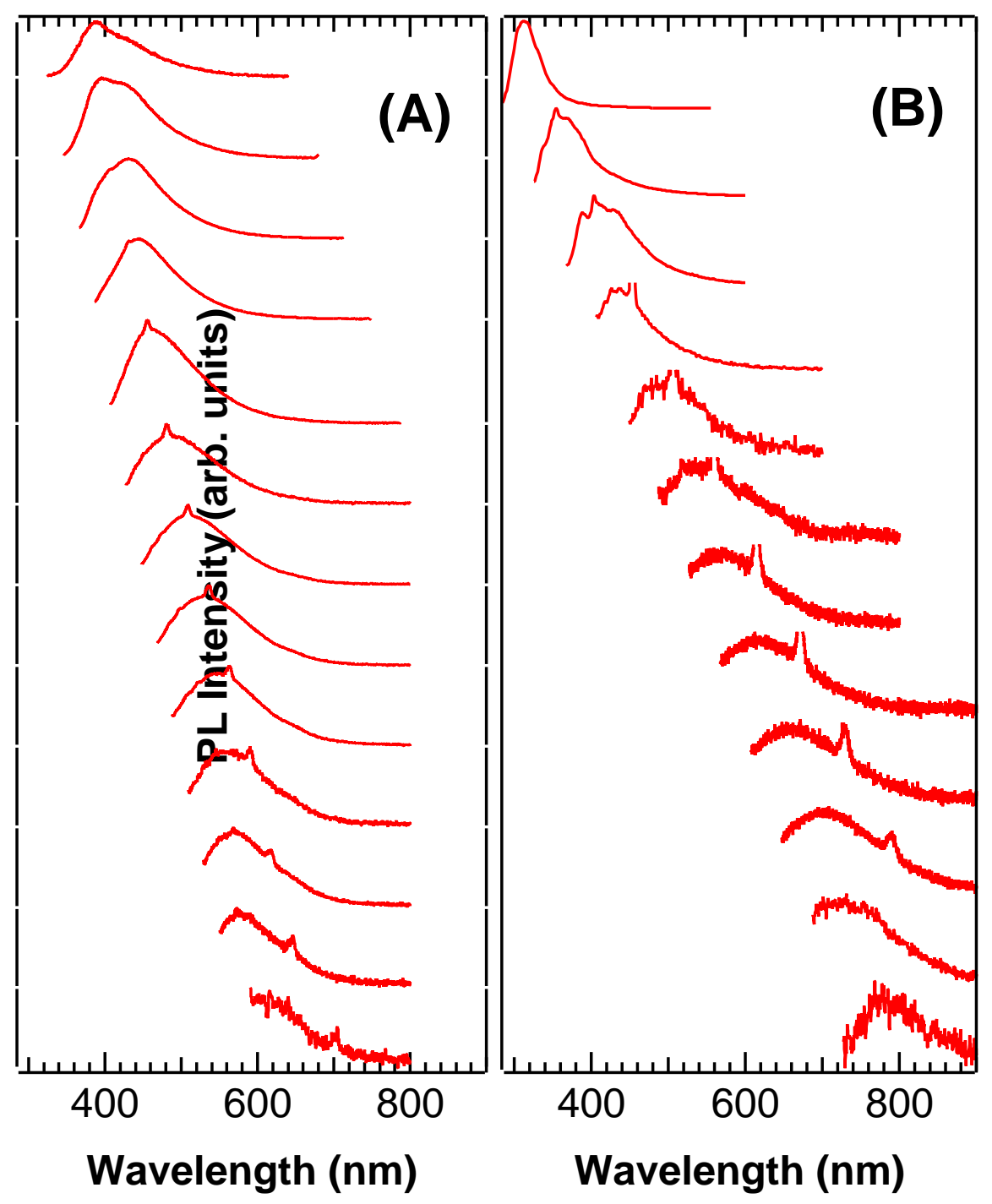

Figure 1. Size-selective photoluminescence (SS-PL) spectra of colloids of (A) oxide terminated and (B) methyl terminated Si quantum dots taken under ambient conditions. The spectra are vertically displaced for clarity. Narrow spectral features result from Raman scattering of the 
excitation light by the $\mathrm{C}-\mathrm{H}$ stretching vibration from the solvent (methanol or hexane).

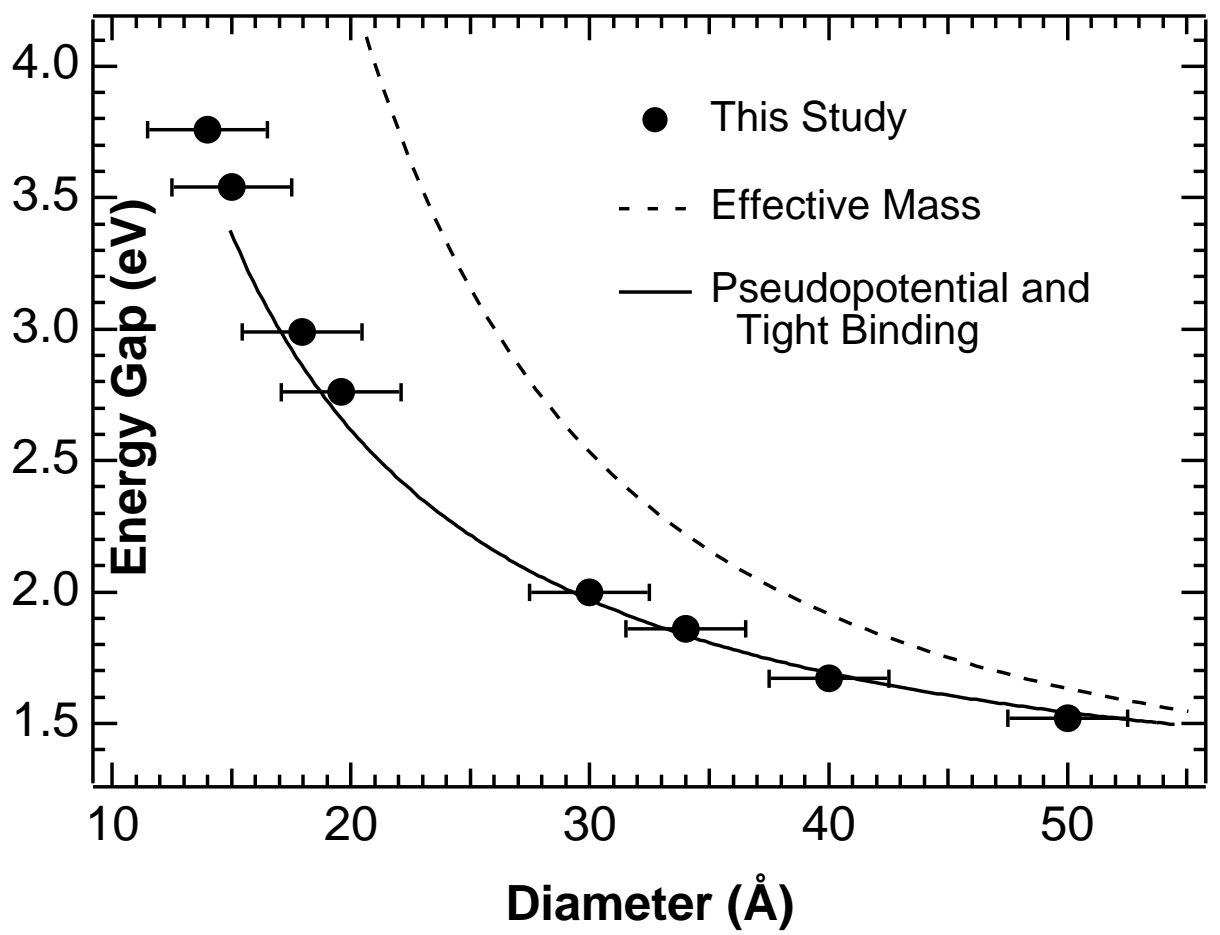

Figure 2. Size dependence of the Si quantum dot energy gap. Our results are compared to effective mass, pseudopotential, and tight binding approaches.

Our results indicate that electroluminescent (EL) devices made with these QDs may also have this broad spectral tunability. Other advantages arising from quantum confinement include high efficiency, long lifetime, reduced sensitivity to traps and defects, simplicity of processing, and lower cost.

In light of these considerations, we developed an extremely versatile active element for use in EL light emitting devices. The active EL material consists of Si or Ge QDs embedded in a thin film ( 1000 ̊) host matrix with a larger bandgap than the energy gap of the QDs (e.g., polyvinylcarbazole, polymethylmethacrylate, silica sol-gels and other organic polymers). Major advantages of this nanocomposite material system are (1) the ease and low cost of producing high quality, thin, conformal EL films by spin coating or vapor deposition (2) the thin film nature of the device also enables conformal and flexible EL devices that may be integrated into a large variety of electronic and optoelectronic circuits or systems, (3) control of the EL color by controlling the size of the QDs, (4) compatibility with mature Si microelectronics, and (5) devices made from these QDs are expected to be robust and long lived because the active material is a robust inorganic material $(\mathrm{Si}, \mathrm{Ge})$ that can tolerate extreme conditions. 
The resultant EL devices operate via electron-hole injection into the active medium followed by radiative recombination of the injected carriers in the active medium. The QDs in the host matrix act as traps or radiative recombination centers within the matrix. The matrix serves several functions (1) a convenient medium to disperse the QDs in the appropriate concentration, (2) a binding medium for the QDs, (3) a matrix that enables low-cost and simple processing of the active EL nanomaterial into thin, flexible and conformal films, (4) an active EL material that may be easily integrated into a large variety of electronic and optoelectronic circuits or systems, and (5) assist in transport of carriers injected into the active EL nanomaterial to the QDs. Electrons are injected into the active medium via a cathode, which is a material with a low work function. A low work function materials is needed since the electron affinity of most materials, in particular organic materials that may comprise the host medium, is on the order of a few volts. Holes are injected via the anode, which is a material with large work function. Various EL device configurations can be used: (1) single layered devices and (2) multi-layered devices. Single layered devices consist of the active medium sandwiched between the cathode and anode. Multi-layered devices are comprised of the active medium and other carrier transport layers between the cathode and anode to assist in the injection of carriers into the active EL material. Forward electrical bias results in light emission. These EL devices perform as efficient and readily tunable EL devices.

This EL device concept was reduced to practice using Si and Ge QDs. Single layered devices were prepared as follows. The anode consisted of patterned Indium-Tin-Oxide (ITO) on a glass substrate. Thin $(\sim 2000 \AA)$ EL films were deposited on the ITO by spin coating. The EL films were comprised of group IV semiconductor QDs (silicon or germanium) embedded in polyvinylcarbazole (PVK) or silica sol-gels. The cathode consisted of evaporated thin (1000 $1500 \AA$ A) films of a low work function material such as $\mathrm{Ca}, \mathrm{Mg}$ or Al. Multi-layered devices were prepared under similar conditions described for the single layered device, but a thin $(\sim 400 \AA)$ electron transport layer (e.g., tris(8-hydroxyquinoline) aluminum) was added between the cathode contact and the EL matrix. These devices produced blue electroluminescence as a result of the small size of the silicon or germanium QDs. The electroluminescnece was consistent with the photoluminescence of the QDs and changed with the size of the QDs in a manner consistent with quantum confinement of carriers in the QDs. The quantum efficiency of these blue emitting prototype devices was $\sim 0.2 \%-0.5 \%$. These QD-LEDs are shown in Figures 3 and 4. 

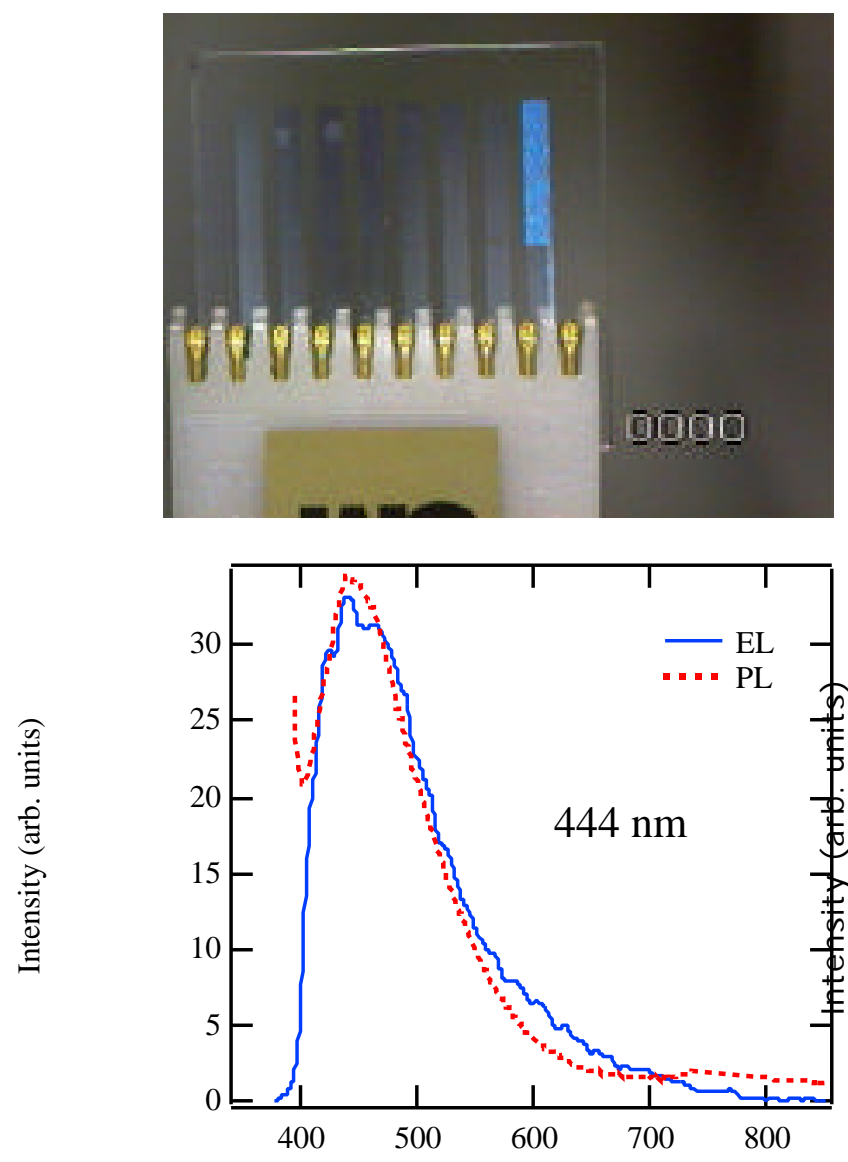

Wavelength (nm)
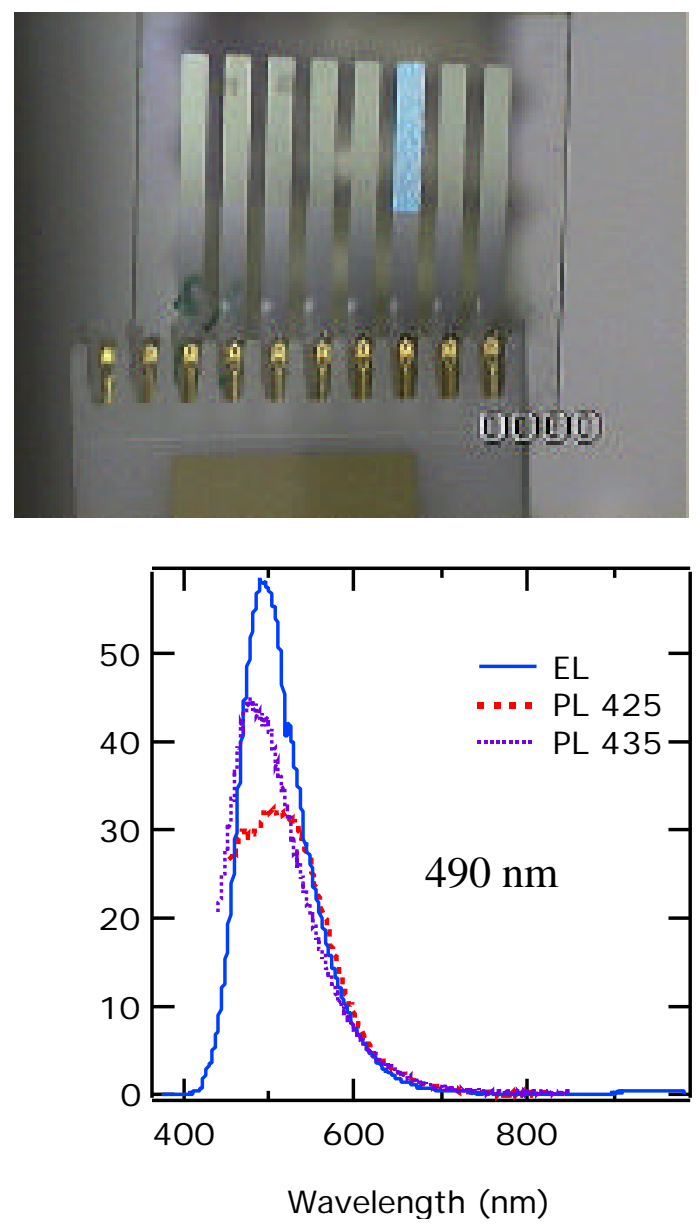

Figure 3. Photograph of an operating blue EL Figure 4. Photograph of an operating blue EL device based on Si QDs in PVK, along with a comparison of the EL and PL spectra. The Si QDs are smaller than those used in the device in Figure 4. device based on Si QDs in PVK, along with a comparison of the EL and PL spectra. The Si QDs are larger than those used in the device in Figure 3.

In addition to the successful demonstration of blue QD-LEDs, we were also interested in exploring the use of semiconductor QDs for blue lasers. In this application, the simple observation of blue emission from the active material is not sufficient. A critical prerequisite for lasing action is the more demanding requirement of optical gain. We performed femtosecond optical gain measurements on colloidal suspensions of Si and Ge QDs and demonstrated the first and only measurement of optical bleaching and gain in these indirect bandgap QDs (Figure 5). This is a critical development and indicates that blue lasers are theoretically possible with these QDs, thus opening the door for a new class of blue and possibly UV lasers with the same advantages as the QD-LEDs we developed. A blue QD laser will enable many future photonics technologies important to both the private sector and DOE missions. 


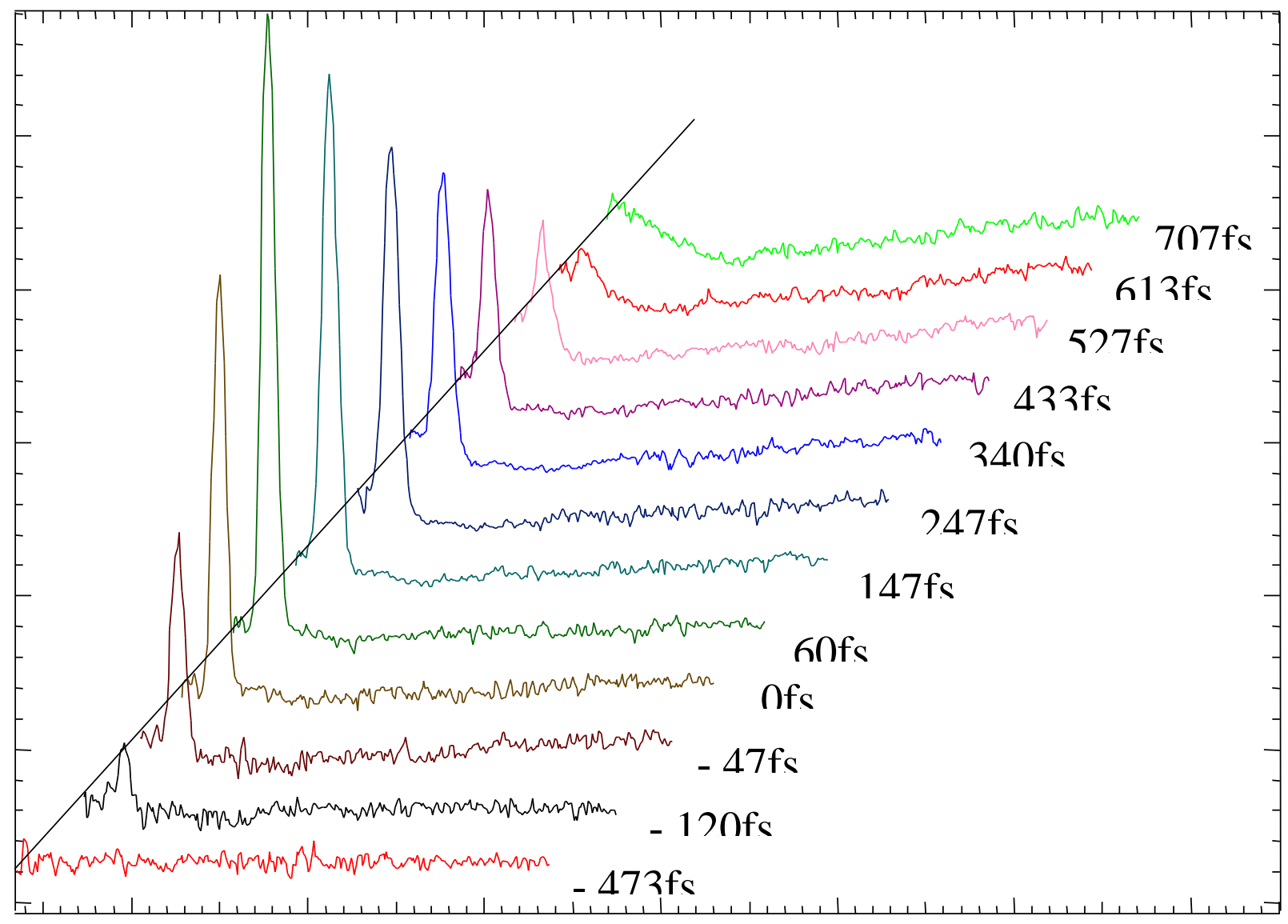

Wavelength (nm)

Figure 5. First demonstration of optical gain from Ge quantum dots. The differential transmission is plotted for various times after optical excitation with a femtosecond pump pulse at $400 \mathrm{~nm}$. The narrow and positive spectral features corresponds to the optical gain. 


\section{Publications}

H. W. H. Lee, P. A. Thielen, G. R. Delgado, S. M. Kauzlarich, C. S Yang, R. A. Bley, D. Mayeri, and B. R. Taylor, "The Origin and Nature of Stable Red to Ultraviolet Light Emission From Silicon Quantum Dots," Science, submitted.

C. A. Smith, H. W. H. Lee, V. J. Leppert, and S. H. Risbud, "Ultraviolet-Blue Emission and Electron-Hole States in ZnSe Quantum Dots," Appl. Phys. Lett, 75, 1688(1999). 